Proc. Indian Acad. Sci. (Chem. Sci.), Vol. 102, No. 5, October 1990, pp. 643-652.

(C) Printed in India.

\title{
Resonance Raman scattering in mercury telluride
}

\author{
M L BANSAL ${ }^{\dagger}$ and ALKA INGALE \\ Nuclear Physics Division, Bhabha Atomic Research Centre, Bombay 400085, India \\ Present address: Laser Programme, Centre for Advanced Technology, Indore 452 012, India
}

\begin{abstract}
HgTe has inverted band structure. Various differences that arise as a consequence of this feature are discussed and it is shown that standard macroscopic formalism for Raman scattering near $E_{1}$ and $E_{1}+\Delta_{1}$ gaps for zincblende structure materials can be applied to $\mathrm{HgTe}$ also. The unusually low value of ODP $d_{30}^{5}$ obtained from Raman measurements is explained in terms of the inverted band structure of $\mathrm{HgTe}$.
\end{abstract}

Keywords. Resonance Raman scattering; mercury telluride; inverted band structure; standard macroscopic formalism.

\section{Introduction}

Light scattering investigation of semiconductors provide important information about various excitations and their interaction. In this work we confine ourselves to Raman intensity variation of TO-phonons. This variation of intensity with laser wavelength, particularly in the neighbourhood of electronic critical points, provides important information about the electron-phonon interaction in terms of band deformation potentials.

Several materials like Ge, Si, GaAs, GaP, InP, InSb, InAs etc. (all cubic) have been investigated extensively (Cardona 1982). Wurtzite type $\mathrm{ZnS}, \mathrm{CdS}, \mathrm{ZnSe}$ etc. have also received considerable attention (Balkanski 1971). The cubics (diamond and zinc-blende structure) have only one triply degenerate optic mode with simple and well-understood electronic band structure. These features allow the exact calculation of Raman cross-section and hence the interest in absolute Raman cross-section measurements. For an excellent review this field the reader is referred to an excellent review by Cardona (1982).

Zero gap semicondetors (semimetals) like HgTe, HgSe have simple zinc-blende structure with inverted band gap (Dornhaus and Nimtz 1980). These have important applications in infrared technology. However, these are relatively less investigated by Raman spectroscopy. In this work we present results on the intensity variation of the TO-phonon (Ingale et al 1989). The plan of presentation is as follows. In $\$ 2$ we briefly summarise the various contributions to the Raman tensor. Section 3 deals with electronic band structure of $\mathrm{HgTe}$ and delineates important differences in its band structure vis $a$ vis that of other well-investigated zinc-blende structures. Here we also discuss some other peculiarities of $\mathrm{HgTe}$. We present the results and their interpretation in $\S 4$. We conclude in $\S 5$ with some suggestions.

\footnotetext{
${ }^{\dagger}$ Deceased
} 


\section{Background theory}

The photon counting rate outside the sample is given by (Cardona 1982; Compaan and Trodahl 1984)

$$
R_{S}^{\prime}=\left(\frac{T_{S} T_{L}}{\left(\alpha_{L}+\alpha_{S}\right) \eta_{L} \eta_{S}}\right) \frac{\omega_{S}^{3} P_{L} \Delta \Omega^{\prime}}{C^{4}} \frac{\left(n\left(\Omega_{p h}\right)+1\right)}{M^{*} \Omega_{p h} v_{c}}\left|\hat{e}_{S} \cdot \boldsymbol{R} \cdot \hat{e}_{L}\right|^{2}
$$

where $P_{L}$ is the incident laser power outside the sample, $T_{S, L}=1-r_{S, L}$ is the fraction of the scattered or incident photons transmitted. The factor $\Delta \Omega^{\prime} / \eta_{L} \eta_{S}$ is the angle divided by refractive index square i.e. the effective collection angle. Factor $1 / v_{c}$ gives the total number of unit cells per unit volume. The factor $\left.\left\{\left[n\left(\Omega_{p h}\right)+1\right)\right] / M^{*} \Omega_{p h}\right\}^{\frac{1}{2}}$ gives the magnitude of displacement due to phonons. The last factor $\left|e_{s} \cdot R \cdot e_{L}\right|$ defines a component of the Raman tensor for the phonon with frequency $\Omega_{p h}$ contracted with incident and scattered polarizations. The independent components of $R$ are called Raman polarizabilities.

The RHS of (1) should be multiplied by another factor called detection efficiency. This factor is a function of wavelength of scattered light and its absolute determination is rather tricky. This however, can be evaluated to within $5-10 \%$ using a sample substitution method (Cardona 1982). One uses various known standards like $\mathrm{Si}, \mathrm{CaF}_{2}$, diamond etc. It may be added here that for opaque samples where back-scattering is employed it is simpler to use $\mathrm{Si}$; even though error margin on its Raman polarizability is large $\left(|a| \simeq 40 \pm 10 \AA^{2}\right.$ at $\omega_{L}=2 \cdot 0 \mathrm{eV}$, Wagner and Cardona 1983).

In diamond and zinc-blende semiconductors the triply degenerate optic phonon has, in the dipole approximation, only one independent Raman polarizability component i.e. $R_{i j}=a\left(1-\delta_{i j}\right)$.

It is the variation of $a$ (Raman susceptibility) which is of interest from the theoretical view point. Raman polarizability near the $E_{1}$ gap in diamond and zinc-blende type compounds is given by (Cardona 1982)

$$
a(E)=\frac{a_{0}^{2}}{4 \sqrt{6}}\left[-\frac{1}{2 \sqrt{2}} d_{10}^{5} \frac{\mathrm{d} \chi(E)}{\mathrm{d} E_{1}}+2 d_{30}^{5} \frac{\left(\chi^{E_{1}}(E)-\chi^{E_{1}+\Delta_{1}}(E)\right)}{\Delta_{1}}+C\right] \ldots,
$$

where $a_{0}$ is the lattice constant and $C$ represents the constant contribution due to other electronic transitions of the medium, assumed far away from $E_{1}$ or $E_{1}+\Delta_{1}$. The first term represents the phonon induced modulation of the $E_{1}$ gap. $d_{10}^{5}$ is the relative change in energies of the conduction and heavy hole valence bands, due to the symmetric part $\left[\hat{\eta}_{p h} \|(111)\right]$ of the $q=0$ phonon (Potz and Vogl 1981). Microscopically this term is essentially a consequence of intraband scattering of electrons (holes) by phonons. Figure 1a shows the two-band scattering process and its contribution to Raman susceptibility can be written as (Martin and Falicov 1975; Pinczuk and Burstein 1975)

$$
\frac{\left\langle 0\left|H_{e R}\right| e_{c} h_{v}\right\rangle\left\langle e_{c} h_{v}\left|H_{e L}\right| e_{c} h_{v}\right\rangle\left\langle e_{c} h_{v}\left|H_{e R}\right| 0\right\rangle}{\left(E_{1}+E_{0}-E\right)\left(E_{1}-E\right)}
$$

where $E, E_{1}$ and $E_{0}$ are the energies of incident photon, $e-h$ pair and the phonon created in the scattering process. This is only qualitative treatment in the sense that, for the actual case that we are discussing, the density of states and variation of 
(a)

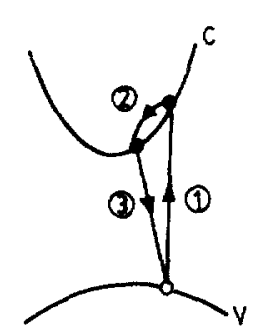

(b)

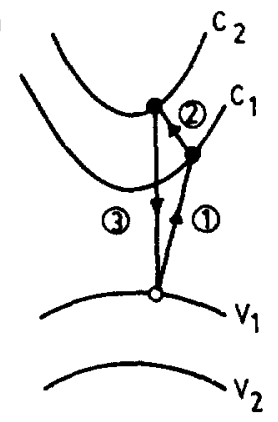

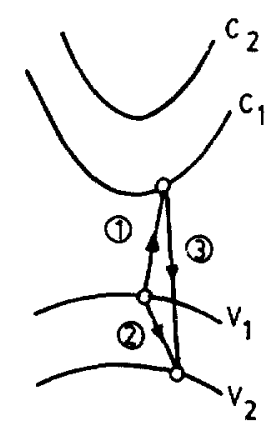

Figure 1. (a) Two band contribution to inelastic light scattering. Step (2) is phonon induced intraband electron (hole) scattering (Pinczuk and Burstein 1975). (b) Three band contribution to inelastic light scattering. In the case of $E_{1}$ and $E_{1}+\Lambda_{1}$ gaps in zinc blende semiconductors, contributions given by the figure on the right are important (Pinczuk and Burstein 1975).

transition energy with $q$ have to be included (Pinczuk and Burstein 1975; Cardona 1982). If in the $e-h$ pair state $e_{c} h_{v}$ the electron and hole are assumed to be uncorrelated, one can write (Pinczuk and Burstein 1975)

$$
\begin{aligned}
\left\langle e_{c} h_{v}\left|H_{e L}\right| e_{c} h_{v}\right\rangle & =\left[d_{10}^{5}(c)-d_{10}^{5}(v)\right],\left\langle u_{\mathrm{rel}} \|(111)\right\rangle \\
& =d_{10}^{5}\left\langle u_{\mathrm{rel}} \mid \mathrm{l}(111)\right\rangle .
\end{aligned}
$$

Neglecting the weak $1 / E$ dependence of matrix elements (3) can be simplified to (Martin and Falicov 1975)

$$
\left[\frac{\chi(E)-\chi\left(E-E_{0}\right)}{E_{0}}\right] d_{10}^{5}
$$

which in the quasistatic limit $E_{0} \rightarrow 0$ reduces to $(\mathrm{d} \chi / \mathrm{d} E) \cdot d_{10}^{5}$ resulting in the most dispersive term for the two-band scattering process.

The second term in (2) is due to three-band scattering. The deformation potential $d_{30}^{5}$ represents the strength of coupling of the two spin-orbit split valence bands $(\mathrm{HH}$ and $L H$ ) due to the $L_{3}$ (at $L$ point) part of the $q=0$ phonon (Potz and Vogl 1981) with $\hat{\eta}_{p h} \perp$ (111). Figure $1 \mathrm{~b}$ shows the three-band scattering process. Qualitatively its contribution can be written as

$$
\frac{\left\langle 0\left|H_{e R}\right| e_{c} h_{2}\right\rangle\left\langle e_{c} h_{2}\left|H_{e L}\right| e_{c} h_{1}\right\rangle\left\langle e_{c} h_{1}\left|H_{e R}\right| 0\right\rangle}{\left(E_{1}+\Delta_{1}+E_{0}-E\right) /\left(E_{1}-E\right)} \ldots
$$

where

$$
\left\langle e_{\mathrm{c}} h_{2}\left|H_{e L}\right| e_{\mathrm{c}} h_{1}\right\rangle=d_{30}^{5}\left\langle u_{\mathrm{rel}} \perp(111)\right\rangle \text {. }
$$

Under the approximation $\left\langle e_{c} h_{2}\left|H_{e R}\right| 0\right\rangle=\left\langle e_{c} h_{1}\left|H_{e R}\right| 0\right\rangle$ (valid if the bands 1 and 2 are spin-orbit split pairs) and small $\Delta_{1}$, it can be shown (Cardona 1982) that this contribution reduces to the second term of (2) viz.

$$
d_{30}^{5}\left(\frac{\chi^{E_{1}}-\chi^{E_{1}+\Delta_{1}}}{\Delta_{1}}\right)
$$

With this heuristic insight into the microscopic origin of the two terms in (2), it is 
clear that the deformation potentials $d_{10}^{5}$ and $d_{30}^{5}$ are sort of averages along the (111) directions. We see in the next section the effect of inverted band structure on the various approximations used for writing (2).

\section{Special features in HgTe}

\subsection{Band structure of $\mathrm{HgTe}$}

The II-VI compounds $\mathrm{HgTe}$ and $\mathrm{HgSe}$ are semimetallic and have the so-called inverted band structure vis a vis other zinc-blende semiconductors like InSb, CdTe etc. (Dornhaus and Nimtz 1980). The band structure of interest is shown in figure 2. Here the conduction band is a consequence of crystal field ( $S-O)$ splitting of a $p$-like $\Gamma_{8}$ state, whereas in GaAs etc. it originates from an s-like $\Gamma_{6}$ state. In HgTe the heavy hole (upper) valence band is a consequence of a split $p$-like state whereas the light hole (lower) valence band originates from an $s$-like $\Gamma_{6}$ state. In InSb the lower two valence bands are a consequence of crystal field splitting of a $p$-like $\Gamma_{8}$ state. However, the symmetry representation of the conduction, $H H$ valence and $L H$ valence bands at the $L$-point remains the same as that in InSb. The quantities $E_{1}$ and $\Delta_{1}$ in $\mathrm{HgTe}$ are quite similar to that in $\operatorname{InSb}\left(E_{1}=1.98 \mathrm{eV}, \Delta_{1} \simeq 0.5 \mathrm{eV}\right.$ for InSb and $E_{1}=2.1 \mathrm{eV}$ and $\Delta_{1} \simeq 0.68 \mathrm{eV}$ for $\mathrm{HgTe}$ ).

From the previous section it is clear that the assumption of equal matrix elements i.e. $\left\langle e_{c} h_{1}\left|H_{e R}\right| 0\right\rangle \simeq\left\langle e_{c} h_{2}\left|H_{e R}\right| 0\right\rangle$ should be valid for one to be able to use the macroscopic expression for Raman susceptibility (2). From susceptibility measurements of Viña et al (1984) it turns out that the above approximation holds even for HgTe implying that the conduction band state at the $L$ point is an admixture of several atom-like states.

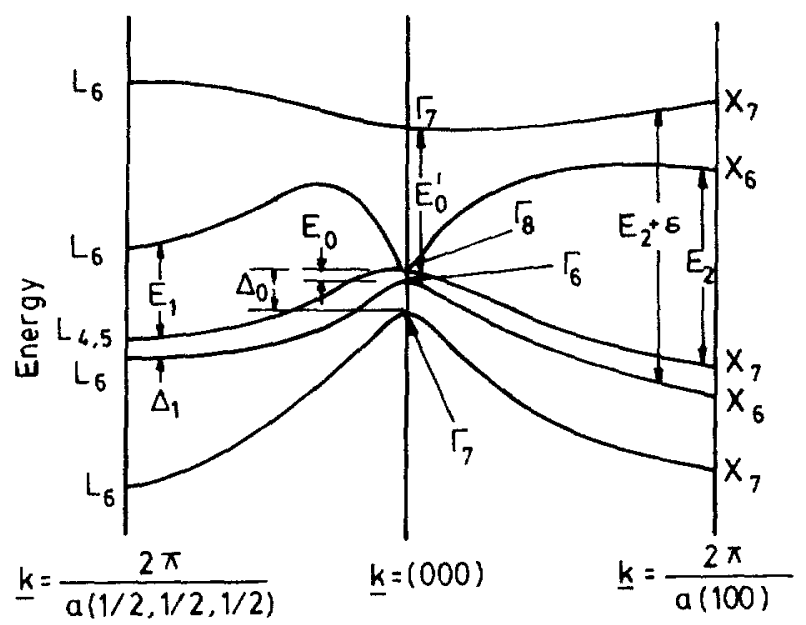

Figure 2. Electronic band structure of $\mathrm{HgTe}$. Notice the origin of the two valence bands $\left(L_{4,5}\right.$ and $\left.L_{6}\right)$ (Dornhaus and Nimtz 1980). 


\subsection{Effects of partial band occupancy}

An additional complication in $\mathrm{HgTe}$ is that, as grown $\mathrm{HgTe}$ samples are p-type because of $\mathrm{Hg}$ vacancies (Dornhauz and Nimtz 1980). The electrons from the valence band are trapped by these vacant sites to render the material p-type at $0 \mathrm{~K}$. As the temperature is raised these trapped electrons are liberated and the Hall coefficient changes its sign. This phenomenon is akin to doping.

In a recent work Sood et al (1985) have studied heavily doped Ge. They find a change of a few percent from the cross-section predicted by (2) in samples with $n_{d}$ $10^{19} / \mathrm{cm}^{3}$. The explanation offered by the authors in terms of inhibited transitions is untenable because this effect is already accounted for in the experimentally measured susceptibility. Pinczuk and Burstein (1971) have pointed out that an additional process of the type shown in figure 3 should also be considered in heavily doped semiconductors with partially filled (vacant) conduction (valence) bands. Here the initial or final electronic transition is intraband.

In HgTe with carrier concentration in the range of $10^{16}-10^{18}, \omega_{p}$ is very small compared to $E_{1}$ and therefore this contribution and hence correction to (2) will be negligible. This in fact is confirmed by the results of Sood et al (1985) where for carrier concentration in the range of $10^{19}$ the departure from (2) is only by a few percent.

\subsection{Phonon damping and interference effects}

Near the zone centre the conduction and valence bands (figure 2) intersect, leading to a continuum of interband electronic transitions from the $H H$ valence band to the conduction band. These excitations have transition energies starting from very near zero for ideal $\mathrm{HgTe}$ (no $\mathrm{Hg}$ vacancies) and the continuum of these transitions overlaps with phonon energy. In the case of the usual p-type $\mathrm{HgTe}$ samples, at finite temperatures, the conduction band and valence bands are partially occupied and empty, respectively. Thus intraband single particle excitations spanning the range $0>w>q_{c} v_{f}\left(q_{c} \simeq 2 \pi k / \lambda ; k\right.$ is the imaginary part of the refractive index and $\lambda$ is the wavelength of light) are in principle possible. For typical $\mathrm{HgTe}$ samples $q_{c} v_{f}$ works out to around $200 \mathrm{~cm}^{-1}$. Thus in $\mathrm{HgTe}$ (or $\mathrm{HgSe}$ ) both or one of the above processes can provide a continuum due to electronic transitions which overlaps with optic-phonon energy. Interference of Raman scattered photons from the discrete (phonon) and continuum (electronic in this case) is known (Klein 1975) to result in Fano-type assymmetric line shape for the optic phonon. This phenomenon is important and observable in situations where the $e-h$ pair states produced near the $\Gamma$-point (through A.p perturbation) provide a dominant scattering channel. Fortunately for

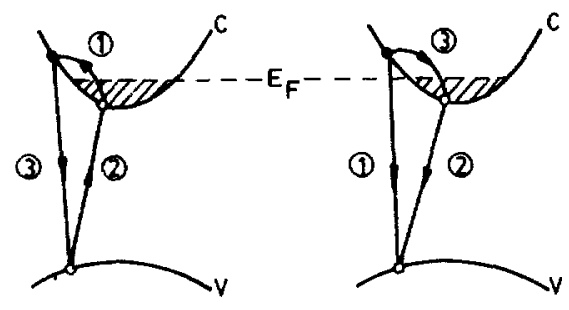

Figure 3. Additional intraband electronic transitions that contribute to scattering in an n-type semiconductor (Pinczuk and Burstein 1975). 
scattering near $E_{1}$ and $E_{1}+\Delta_{1}$ gaps in $\mathrm{HgTe}$, this complication is avoided because the $E_{0}+\Delta_{0}$ and other gaps at the $\Gamma$ - point are far removed in energy from these gaps $\left(E_{1}\right.$ and $\left.E_{1}+\Delta_{1}\right)$. Moreover the large $q$ difference $\left[\pi / a_{0}(111)\right]$ between $L$ and $\Gamma$-points rules out the possibility of $e-h$ pairs at the $L$ point contributing to scattering due to electronic excitations at $\Gamma$-point.

Thus we see that in spite of the several differences that are there in $\mathrm{HgTe}$ due to inverted band structure vis a vis that of InSb, (2) remains valid and can be used for obtaining deformation potentials from absolute Raman intensity measurements.

\subsection{Surface characteristics}

$\mathrm{HgTe}$ is a soft material and mechanical polish alone is not sufficient to yield a good surface for Raman or linear susceptibility measurements. A chemo-mechanical polish $\left(0.01 \% \mathrm{Br}_{2}\right.$ in $\left.\mathrm{CH}_{3} \mathrm{OH}\right)$ followed by free etch in the same solution results in a good surface for reproducible Raman measurements. There are indications that differences exist in $\mathrm{Hg}$ to $\mathrm{Te}$ content near the surface in $\mathrm{HgTe}$ subjected to a different etching procedure (Rhiger and Kvaas 1982). Viña et al (1984) however, find no evidence of a damaged Te-rich layer after $\mathrm{Br}_{2}(0.01 \%)+\mathrm{CH}_{3} \mathrm{OH}$ etching.

\section{Results and discussion}

\subsection{Raman susceptibility for $\mathrm{HgTe}$}

Using the data of Viña et al (1984) the factor $T_{S} T_{L} /\left[\left(\alpha_{L}+\alpha_{S}\right) \eta_{L} \eta_{S}\right]$ in (1) can be evaluated. This factor, as shown in figure 4 , is about four times smaller than that encountered in Ge (Sood et al 1985), leading to weaker Raman signal for HgTe.

In order to obtain absolute Raman susceptibility by the sample substitution method, (111) silicon has been employed. We find that an expression of the type

$$
a(\mathrm{Si})=70 /\left(\omega_{g}-\omega\right)^{3 / 2} \AA^{2},
$$

can be used for Raman susceptibility of silicon and in fact gives a better fit over the range 1.8 to $3 \mathrm{eV}$ for various data values found in literature (Wagner and Cordona 1983; Compaan and Trodahl 1984). It must be mentioned here that the factor 70 itself has a large error bar $( \pm 20 \%)$ (Menendez 1985).

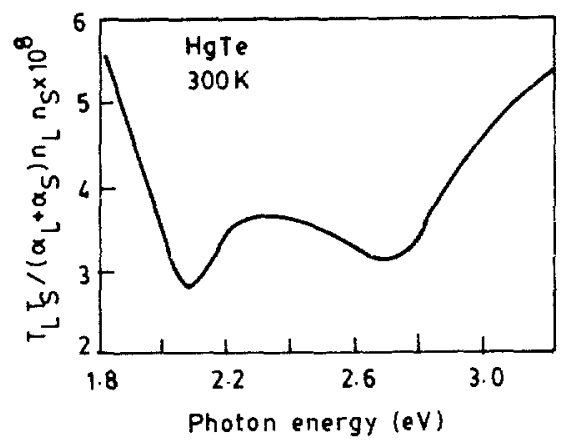

Figure 4. Correction factor at room temperature for $\mathrm{HgTe}$ based on ellipsometric measurements of Viña et al (1984). 


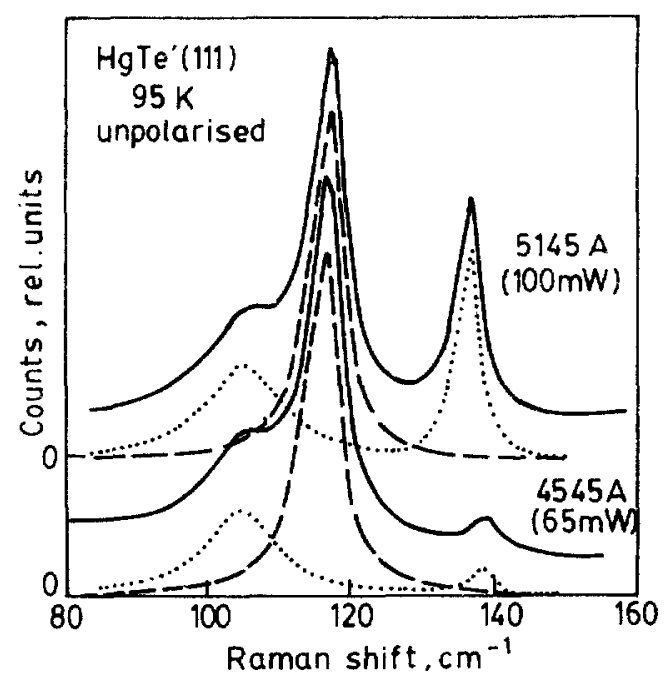

Figure 5. Typical Raman spectra at two different wavelengths. Intensity for the $117 \mathrm{~cm}^{-1}$ TO-phonon was obtained through least squares fit. The resolved TO-phonon peaks are shown as smooth lorentzians.

Raman measurements have been performed on the (111) face of $\mathrm{HgTe}$ at $95 \mathrm{~K}$ without any polarization analysis of the scattered light. In this configuration one obtains TO, LO and forbidden LO modes with intensities proportional to (4/3) $a_{T}^{2}$, $(1 / 3) a_{L}^{2}$ and $a_{F}^{2}$ respectively.

In figure 5 we show the typical spectra recorded at two different laser energies. Least squares fit is used to separate the three peaks that are seen in figure 5 . The origin of the lowest frequency peak is still not clear in spite of a number of speculations and we do not discuss it here. The large intensity and slightly lower frequency of the LO-peak (5145 A spectra) is consistent with its being a dominantly forbidden LO at this wavelength. Using (1) and after taking care, of various factors, the obtained Raman susceptibility squared is plotted in figure 7 below.

For theoretical evaluation of Raman susceptibility using (2), we use the ellipsometric measurements of Viña et al (1984) for $\mathrm{HgTe}$ at room temperature. Their data can be fitted (Viña et al 1984; Ingale et al 1990) using analytical expressions for the $E_{1}$ and $E_{1}+\Delta_{1}$ critical points. The values of the amplitudes obtained are in reasonable agreement with theoretical (Cardona 1982) values for the $E_{1}$ and $E_{1}+\Delta_{1}$ critical points, in normal semiconductors like InSb, justifying the assumption discussed earlier in $\S 3 \cdot 1$. The functional form of $\chi$ along with $\chi^{E_{1}}$ and $\chi^{E_{1}+\Delta_{1}}$ contributions has been used in (2) to obtain the shape of resonance curves as a function of ratio $d_{30}^{5} / d_{10}^{5}$, as shown in figure 6 . Various curves in this figure have been scaled suitably in order to bring out the shape differences more clearly. The strong dependence of shape on the parameter $d_{30}^{5} / d_{10}^{5}$ is indicative of similar magnitude of the two-band (first) and three-band (second) terms in (2). This is a consequence of rather large Lorentzian broadening of the $E_{1}$ and $E_{1}+\Delta_{1}$ critical points in this compound (Viña et al 1984).

Figure 7 shows the experimental Raman susceptibility squared plotted against the laser wavelength (Ingale et al 1990). The theoretical curve corresponds to $d_{30}^{5} / d_{10}^{5}=$ $-0 \cdot 8$, with $\left|d_{30}^{5}\right|=14$ and $\left|d_{10}^{5}\right|=18 \mathrm{eV}$. The critical point energies $E_{1}$ and $E_{1}+\Delta_{1}$ 


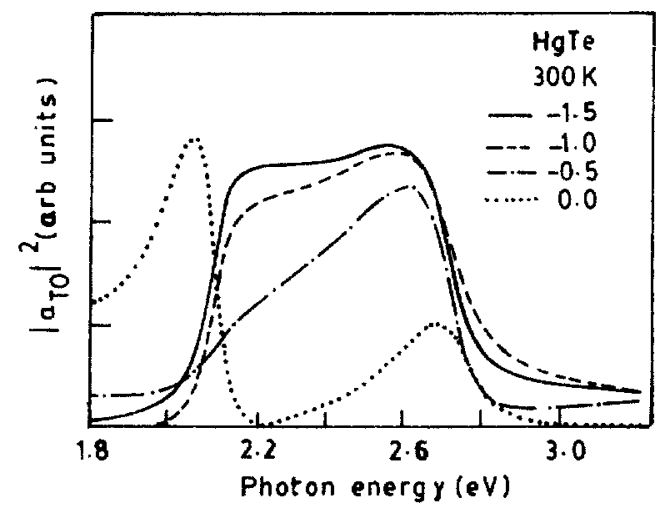

Figure 6. Shape of resonance curves at different values of parameter $d_{30}^{5} / d_{10}^{5}$. The value 0 for this parameter corresponds to two band terms alone.

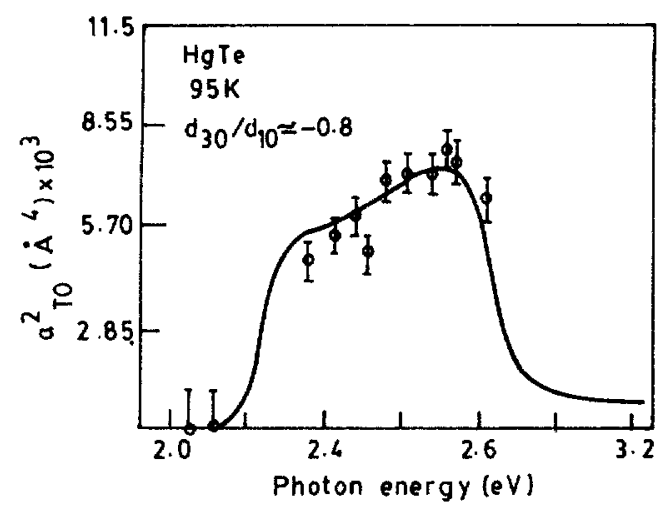

Figure 7. Measured resonance in the Raman polarizability of $\mathrm{HgTe}$ for the allowed TO scattering at $95 \mathrm{~K}$. The solid curve is a calculation using (2) with $\left|d_{30}^{5}\right|=14 \mathrm{eV}$ and $\left|d_{10}^{5}\right|=18 \mathrm{eV}$.

have been shifted from their room temperature values by $0.12 \mathrm{eV}$, to account for the temperature difference $\left[\mathrm{d} E_{1} / d \mathrm{~T} \simeq-0.5-0.6 \times 10^{-4} \mathrm{eV} / \mathrm{K}\right.$ (Rodzik and Kisiel 1983), $\Delta_{1}=$ constant and $\left.\Delta T \simeq 205 \mathrm{~K}\right]$. Here we must stress that the accuracy of the ratio $d_{30}^{5} / d_{10}^{5}$ so obtained is always higher than that of the individual deformation potentials. This follows from the large error bar that is associated with the cross-section for silicon. It is worthwhile noticing that the Raman susceptibility squared $\left(a_{T}^{2}\right)$ obtained here is about a factor of 100 less than that obtained for Ge (Sood et al 1985) and about a factor of 10 less as compared to that in InSb (Menendez et al 1985). This small value of $a_{T}^{2}$ makes measurements difficult in $\mathrm{HgTe}$.

\subsection{Optical deformation potentials}

No theoretical calculations are available for the optical deformation potentials in $\mathrm{HgTe}$. Therefore the only comparison that can be made is with other zinc-blende materials (Cardona 1982). As has been mentioned earlier, InSb has similar energies 
$E_{1}$ and $E_{1}+\Delta_{1}$. Recently Menendez et al (1985) have obtained $d_{10}^{5} \simeq-16 \mathrm{eV}$ and $d_{30}^{5} \simeq 33 \mathrm{eV}$, for InSb. In fact most of the tetrahedral semiconductors for which calculations have been performed have $d_{10}^{5}$ values ranging from $10 \mathrm{eV}$ to $20 \mathrm{eV}$ (Potz and Vogl 1981)* and these are expected to be weakly dependent upon chemical constituents. In view of this the value of $d_{10}^{5}$ obtained for $\mathrm{HgTe}$ is reasonable.

Theoretical values of ' $d_{30}^{5}$ however, are all around $40 \mathrm{eV}$. This is borne out experimentally for $\mathrm{Ge}(46 \mathrm{eV})^{1}, \mathrm{GaAs}(37 \mathrm{eV})$ (Cardona 1982) and InSb $(33 \mathrm{eV})$ (Menendez et al 1984). It is clear that the value of $d_{30}^{5}$ obtained by us for $\mathrm{HgTe}$ is a factor of three less than that for other zinc-blende materials. Such a small value of $d_{30}^{5}$ for $\mathrm{HgTe}$ can be explained if one recalls that $d_{30}^{5}$ represents mixing of $H H$ and $L H$ valence bands (see $\S 2$ ). Both these valence bands originate from a $p$-like atomic state in case of normal zinc-blende semiconductors. HgTe, however, has inverted band structure as a consequence of which the $H H$ valence band originates from a $p$-like atomic state and the $L H$ (lower) valence band originates from an $s$-like atomic state. Thus at $L$-point one would expect the phonon to cause only a small mixing of these two states. We believe, therefore, that the small value of $d_{30}^{5}$ that we have obtained for $\mathrm{HgTe}$ is a direct consequence of the inverted band structure of $\mathrm{HgTe}$. Similar behaviour should be expected in $\mathrm{HgSe}$, which also has inverted band structure.

\section{Conclusions}

We believe that in spite of inherent difficulties (low cross-section, sample decomposition under laser heating at ambient temperature etc.) $\mathrm{HgTe}$ provides an interesting situation where both the two-band and three-band terms are equally important. This feature results in very strong dependence of the shape of the resonance curve upon the ratio $d_{30}^{5} / d_{10}^{5}$. A systematic variation of the band ordering should occur in the mixed system $\mathrm{Hg}_{1-x} \mathrm{Cd}_{x} \mathrm{Te}$. It should be of considerable interest to examine the behaviour of the optical deformation potentials related with $\mathrm{HgTe}$-like and $\mathrm{CdTe}$-like phonons.

\section{Acknowledgements}

Authors are grateful to Dr A P Roy for several helpful discussions. We thank Prof A $\mathrm{K}$ Ramdas for HgTe samples used in the experiments.

\section{References}

Balkanski M (ed.) 1971 Light scattering in solids (Paris: Flammarion)

Cardona M 1982 in Light scattering in solids II: Topics in applied physics (eds) M Cardona and G Gun therodt (Berlin: Springer) vol 50

Compaan A and Trodahl H J 1984 Phys. Rev. B29 793

\footnotetext{
* Values of $d_{10}^{5}$ given in table 2.10 (p. 157) of Cardona (1982) are actually values of $d_{10}^{5}(L H)$ taken from Potz and Vogl (1971). Correct values given by $\left[d_{10}^{5}\left(\right.\right.$ cond) $-d_{10}^{5}$ (val)] can be calculated from table IV of Potz and Vogl (1971). Correct values of $d_{10}^{5}$ have, however, been used in table 2.10 of Cardona (1982) for evaluating $d_{30}^{5} / d_{10}^{5}$, etc.
} 
Dornhaus R and Nimtz G 1980 in Narrow-gap semiconductors: Springer tracts in modern physics (ed.) G Hohler (Berlin: Springer)

Grynderg M, Toullec R Le and Balkanski M 1974 Phys. Rev. B9 517

Ingale A, Bansal M L and Roy A P 1989 Phys. Rev. B40 12353

Klein M V 1975 in Light scattering in solids: Topics in applied physics (ed.) M Cardona (Berlin, Heidelberg, New York: Springer) vol. 8, p. 147

Martin R M and Falicov L M 1975 in Light scattering in solids: Topics in applied physics (ed.) M Cardona (Berlin, Heidelberg, New York: Springer) vol. 8, p. 79

Menendez J, Viña L, Cardona M and Anastassakis E 1985 Phys. Rev. B32 3966

Pinczuk A and Burstein E 1971 in Light scattering in solids (ed.) M Balkanski (Paris: Flammarion)

Pinczuk A and Burstein E 1975 in Light scattering in solids: Topics in applied physics (ed.) M Cardona (Berlin, Heidelberg New York: Springer) vol. 8

Potz W and Vogl P 1981 Phys. Rev. B24 2025

Rhiger D R and Kvaas R E 1982 J. Vacuum Sci. Technol. 21168

Rodzik A and Kisiel A 1983 J. Phys. C(4B)16 203

Sood A K, Contreras G and Cardona M 1985 Phys. Rev. B31 3760

Viña L, Umbach C, Cardona M and Vodopyanov L 1984 Phys. Rev. B29 6752

Wagner J and Cardona M 1983 Solid State Commun. 48301 